\title{
On The Modified Monotonic Loading Concept for the Calculation of the Cyclic J-Integral
}

\section{Ross Beesley}

Department of Mechanical \& Aerospace Engineering, University of Strathclyde, Glasgow, G1 1XJ, UK

\author{
Haofeng Chen ${ }^{1}$ \\ Department of Mechanical \& Aerospace Engineering, University of Strathclyde, Glasgow, \\ G1 1XJ, UK
}

\section{Martin Hughes}

Siemens Industrial Turbomachinery Ltd, Waterside South, Lincoln, LN5 7FD

\begin{abstract}
This paper investigates an approach for calculating the cyclic J-Integral through a new industrial application. A previously proposed method is investigated further with the extension of this technique through a new application of a practical 3D notched component containing a semi-elliptical surface crack. Current methods of calculating the cyclic J-Integral are identified and their limitations discussed. A modified monotonic loading concept is adapted to calculate the cyclic J-integral of this 3D Semi Elliptical Surface Crack under cyclic loading conditions. Both the finite element method (FEM) and the Extended Finite Element Method (XFEM) are discussed as possible methods of calculating the cyclic J-Integral in this investigation. Different loading conditions including uniaxial tension and out of plane shear are applied, and the relationships between the applied loads and the cyclic J-integral are established. In addition, the variations of the cyclic J-integral along the crack front are investigated. This allows the critical load that can be applied before crack propagation occurs to be determined as well as the identification of the critical crack direction once propagation does occur.

These calculations display the applicability of the method to practical examples and illustrate an accurate method of estimating the cyclic J-integral.
\end{abstract}

Keywords: crack, J-integral, cyclic J-integral, fracture mechanics, FEA, XFEM

\footnotetext{
${ }^{1}$ Corresponding author

Email: haofeng.chen@strath.ac.uk
} 


\section{NOMENCLATURE}

Dowling and Begley Fatigue Law Constant

C

Paris' Law Constant

$d a$

Change in crack length

$d N$

Change in number of cycles

J

J-Integral

$\Delta J$

Cyclic J-Integral

Jmax J-Integral at maximum cyclic load

Jmin J-Integral at minimum cyclic load

K

Stress Intensity Factor

$\Delta K$

Stress Intensity Factor range

Kmax Stress Intensity Factor at maximum cyclic load

Kmin Stress Intensity Factor at minimum cyclic load

m Paris' Law Constant

MPa Mega Pascals

N Newtons

$\varepsilon \quad$ Strain

$\sigma \quad$ Stress 


\section{INTRODUCTION}

Fracture mechanics regards the initiation and propagation of cracks. The impact of material fracture varies depending on the specific application but the results can be catastrophic. Therefore, gaining an understanding of fracture and failure is very important. The ability to predict when a crack will initiate and fail, and thus the resulting fatigue life of the component must be understood to ensure the safe design and utilisation of structural components. Fracture mechanics provides generalized techniques that are widely applied to a number of different industries and applications. For this reason, this field of study has attracted a large number of researchers [1-6].

Stress raisers are of particular importance when considering engineering components. Design features such as notches or sharp corners, and even minor defects such as scratches and corrosion can introduce stress raisers which reduce the critical stress at which crack initiation can occur. Such features can limit the fatigue life since failure can occur at a reduced load or fewer loading cycles. The stress intensity factor (SIF), is a measure of the stress conditions near a crack tip and can be used to predict stress and fracture behaviour under different loading conditions. Extensive experimental testing has permitted the development of a set of standardized equations for calculating the stress intensity factor for a number of different crack and model geometries.

Crack simulation is vitally important and there are a number of methods of using fracture mechanics in order to evaluate fracture and fatigue life including R5 and R6 codes $[7,8]$ and stress intensity factor analysis. However, the focus of this paper is the J-Integral, an alternative to SIF when considering elastic plastic fracture mechanics, and how this can be extended to allow the evaluation of fatigue life. Although a more complex parameter than the stress intensity factor, the development of commercial and bespoke finite element software packages has made the J-Integral a more promising technique and as a result, interest in the field has increased in recent years[9-12]. The importance of this as a fracture mechanics and crack simulation parameter is discussed in this paper. Following discussion of the relevant methods of fracture mechanics, the J-Integral is introduced and methods of the extension of this parameter to allow for 
cyclic fatigue are investigated through the application of techniques on an industrial test specimen.

\subsection{Objectives}

The overarching aim of this investigation is to assess the suitability of an extended monotonic analysis for approximating the cyclic J-integral $(\Delta \mathrm{J})$. Initially, the limitations with the current methods of determining the cyclic J-Integral will be identified. The suitability of the proposed Modified Monotonic Loading (MML) concept will then be assessed. Finally, this technique will be applied to an industrial test specimen in order to calculate the cyclic J-Integral and its variation with increasing load and crack front location. This paper is organised as follows:

Section 2 discusses the background theory of numerical methods that exist for addressing crack modelling. In Section 3, finite element methods are introduced and the proposed concept for calculating the $\Delta \mathrm{J}$ will be discussed. Section 4 presents the model specific to this application and the associated material and loading properties defined. The investigation continues with Section 5 which presents the obtained results for the validation of the MML technique as well as the calculated cyclic J-Integral variation with increasing load and crack location.

\section{THEORETICAL BACKGROUND}

\subsection{Contour Integrals}

Contour Integration allows the evaluation of integrals along certain paths[13]. Within finite element, contour integration allows the calculation of fracture mechanics parameters of a material including the stress intensity factor, $\mathrm{K}$, and the J-Integral, J. The finite element software package, ABAQUS[14], allows the calculation of such integrals along paths encircling a crack front. While these contour integrals do not themselves directly predict how a crack will propagate, they can be used to provide valuable information and offer some indication as to how the crack may behave as well as the estimation of fatigue life. 


\subsection{Fatigue Life}

In order to calculate the fatigue life under Linear Elastic Fracture Mechanics (LEFM), the stress intensity factor, $\mathrm{K}$, is required. The SIF is a function of stress and crack length. Under cyclic loading conditions, the SIF range between maximum and minimum loading can be used to predict fatigue life through Paris' Law[15] as shown in Eq 1.

$$
\frac{d a}{d N}=C \Delta K^{m}
$$

Where $a$ is crack length, $N$ is number of cycles, $\Delta K=K_{\max }-K_{\min }$ is the stress intensity factor range and $C$ and $m$ are constants. This relationship holds for LEFM properties, however it does not apply to Elastic-Plastic Fracture Mechanics (EPFM) properties since the relationship becomes less accurate as plasticity levels increase. Therefore, a new parameter is required to allow a similar approach for calculating the fatigue life of

elastic-plastic materials. A suitable alternative is the J-Integral, which represents a method of calculating the strain energy release rate per unit area of a fracture surface. The J-Integral offers an EPFM equivalent to the SIF for LEFM. Calculating the J-Integral under monotonic loading conditions is relatively simple. It is done routinely and has proven itself to be a good method of modelling crack behaviour. However, difficulties arise when implementing cyclic loading conditions.

The cyclic J-Integral, is a function of the stress and strain range, $\Delta \sigma$ and $\Delta \varepsilon$ and as a result, unlike the cyclic stress intensity factor, is not simply equal to $J_{\max } J_{\min }$ and therefore $\Delta J \neq J_{\max }-J_{\min }$. For this reason, calculating the cyclic J-Integral is inherently more difficult than for monotonic loading and no standard techniques have yet been developed to determine the cyclic J-integral. In order to determine the EPFM fatigue life, the J-Integral must be extended to allow for cyclic loading conditions, much like the SIF range is used in LEFM cyclic fatigue.

The J-Integral is a measure of the elastic and plastic work done for crack growth to occur and as a result is assumed to be equal to the sum of the elastic and plastic components, as introduced by Sumpter and Turner[16]. At low levels of plasticity, J is 
dominated by the elastic component and so the linear elastic based strain energy release rate is sufficient for calculating J. However, when the effect of the plastic zone becomes more substantial, this linear elastic approximation is no longer valid. Therefore, since the J-Integral can be used to determine the fatigue life, a reliable method of its calculation is vitally important.

The cyclic J-Integral was first proposed and implemented by Dowling and Begley[17]. A power law behaviour, similar to that of the Paris equation was developed and so the fatigue crack growth rate of cyclic loading EPFM can be written as:

$$
\frac{d a}{d N}=A(\Delta J)^{m}
$$

where $A$ and $m$ are material constants.

\subsection{Limitations of Existing Technologies}

The GE/EPRI[18] and Reference Stress Method (RSM)[19] offer simplified methods of approximating the cyclic J-Integral. However, due to the nature of these methods they exhibit considerable limitations and thus produce overly conservative results. Both of these methods are based on the limit load analysis and as such do not consider the crack geometry, meaning that they are unable to assess three dimensional detail. Consequently, the $\Delta \mathrm{J}$ variation along the crack front cannot be determined and thus valuable crack propagation information is neglected. In addition, these methods are suitable for a number of documented test cases such as compact tension specimens, however, difficulties arise when applying these methods to bespoke specimens.

These limitations provide great approximations in the calculation of the cyclic J-Integral, significantly reducing the accuracy of the results. For these reasons, these methods are not considered appropriate for complex 3D industrial applications.

ABAQUS and other FE packages are capable of calculating the J-Integral under monotonic loading, however, they are currently unable to automatically determine the cyclic J-Integral from stress and strain history. Manually calculating the cyclic J-Integral, 
would require extensive and very time consuming post processing of the analysis history data. Manually calculating in this way is therefore not feasible and so a more automated method is required if the cyclic J-Integral is to be viable fatigue life parameter.

\subsection{Crack Modelling}

Simulating a 3D surface crack is much more complicated than a 2D crack. The variation of $\Delta \mathrm{K}$ and $\Delta \mathrm{J}$ along the crack front is dependent on the type and magnitude of the applied loading and as a result will vary depending upon the location along the 3D surface crack front. Different locations will result in different values of $\Delta \mathrm{K}$ and $\Delta \mathrm{J}$ and will thus affect the crack propagation direction. It is therefore vitally important to simulate the $\Delta \mathrm{J}$ with a high level of detail under different loading conditions in order to gain an understanding of the crack behaviour and thus predict its propagation.

\section{NUMERICAL METHOD}

Modelling simplified models such as infinite plates and blocks can provide valuable insight into crack behaviour. However, these large simplifications can overlook the complexities of real life applications that are found in industry. Therefore, increasing the model complexity makes computational models more akin to industrial applications and thus the results can offer more value than that of greatly simplified cases. This provides reason for modelling a complex geometry test specimen in this investigation.

\subsection{Finite Element Analysis (FEA) Crack Simulation}

FEA allows the modelling of cracks and the calculation of their associated parameters such as SIF and J-Integral. Within ABAQUS, cracks can either be modelled using the traditional Finite Element Method (FEM) or the Extended Finite Element Method (XFEM) [20].

FEM crack modelling requires the definition of contour integral crack properties which allow the crack front and the crack extension direction to be specified. In addition, a seam crack must be implemented which allows the fracture surfaces to 
separate. This method currently only offers modelling of stationary cracks, meaning that propagation cannot automatically be modelled unlike XFEM. Within FEM, mesh quality around the crack tip is critical and must be refined sufficiently to allow for acceptable accuracy which increases the computational modelling and analysis effort.

\subsection{Extended Finite Element Method (XFEM)}

The extended finite element method is capable of modelling mesh independent cracking, meaning that crack initiation and propagation can be modeled without prior definition. A propagating crack does not need to adhere to element boundaries unlike the traditional finite element method (FEM). This reduces the importance of mesh refinement in the region of the crack front. XFEM can model stationary or propagating cracks, however ABAQUS is currently only capable of determining crack parameters such as SIF and J-Integral for stationary cracks. This method is still in its infancy but it shows a great deal of potential. It provides a simple method of modeling complex crack geometries without the need for extensive mesh refinement which can be very computationally expensive both in implementation and analysis. This method is capable of calculating contour integrals such as the SIF and J-Integral, however, when a high level of geometrical detail is introduced, the accuracy of contour integration close to the crack tip is compromised. For this reason, XFEM will not be used as a technique for calculating contour integrals in this investigation and traditional FEM will be used instead.

\subsection{Modified Monotonic Loading (MML) Concept}

A concept has been proposed which provides a reasonable approximation for the calculation of the cyclic J-Integral which addresses the known issues in the existing technologies. This can be achieved through modification of a monotonic loading analysis by replacing $\sigma_{y}$ with $2 \sigma_{y}$ and replacing the cyclic load range with a single monotonic load equal to the range. This allows such a modified monotonic analysis to replicate the conditions of a cyclic loading analysis. This method is referred to in this paper as the 
Modified Monotonic Loading (MML) concept. This follows on from the work of Chen and Chen[21]. It was discovered that in an un-cracked body subjected to variable loading conditions, the differences between this MML concept and the equivalent cyclic analysis were relatively small. Their work indicated the potential for this technique as a method of determining the cyclic J-Integral. In this investigation, this MML concept will be investigated and tested further on an industrial test specimen.

Rice[3] originally defined the J-Integral in two dimensions as:

$$
J=\int_{\Gamma}\left(W d y-T \frac{\partial u}{\partial x} d s\right)
$$

Where $\mathrm{T}$ is the traction vector, $\mathrm{u}$ is the displacement vector, $\mathrm{ds}$ is an element of arc length along the contour path, $\Gamma$, and $W$ is the strain energy density which is given by:

$$
W=\int_{0}^{\varepsilon} \sigma_{i j} d \varepsilon_{i j}
$$

Where $\varepsilon$ is an infinitesimal strain tensor.

Tanaka [22] later extended this for cyclic loading between two states, i and j, as:

$$
\Delta J_{j / i}=\int_{\Gamma}\left(\Delta W d x_{2}-\Delta T_{m} \frac{\partial \Delta u_{m}}{\partial d x_{1}} d s\right)
$$

Altering the terminology to match Rice's original equation, allows it to be represented as:

$$
\Delta J=\int_{\Gamma}\left(\Delta W d y-\Delta T \frac{\partial \Delta u}{\partial d x} d s\right)
$$

Where $\Delta \mathrm{W}$ is the strain energy density range between two states, $\mathrm{i}$ and $\mathrm{j}$, which is a function of the stress and strain range and is given by:

$$
\Delta W=\int_{\left(\varepsilon_{m n}\right)_{i}}^{\left(\varepsilon_{m n}\right)_{j}}\left[\sigma_{k l}-\left(\sigma_{m n}\right)_{i}\right] d \varepsilon_{k l}
$$

Where $\sigma_{k l}$ and $\varepsilon_{k l}$ are the stress and strain tensors respectively and $\sigma_{m n}$ and $\varepsilon_{m n}$ denote relative changes between values corresponding to the load change from one state to another.

It can therefore be seen that the J-Integral is a function of stress and strain, and the cyclic J-Integral is a function of the stress range and strain range as illustrated in Eq. (8) and Eq. (9). Therefore, for this concept to be viable and the hypothesis that it is capable of accurately replicating a cyclic loading analysis to hold true, then the stress and strain 
data from the modified monotonic loading analysis must match the stress range and strain range data from a cyclic loading analysis, Eq. (10) \& Eq. (11).

Using this assumption will then allow the determination of the cyclic J-Integral through the MML concept within ABAQUS. Following such a hypothesis, the cyclic J-Integral values under fatigue loading can be assumed to be equal to the J-Integral values from the Modified Monotonic Loading analysis, Eq. (12). It is assumed that:

$$
\begin{aligned}
& J=f(\sigma, \varepsilon) \\
& \Delta J=f(\Delta \sigma, \Delta \varepsilon)
\end{aligned}
$$

Therefore, if

$$
\sigma_{M M L}=\Delta \sigma_{c y c l i c}
$$

And

$$
\varepsilon_{M M L}=\Delta \varepsilon_{\text {cyclic }}
$$

Then,

$$
J_{M M L}=\Delta J_{\text {cyclic }}
$$

Therefore, for a cyclic loading analysis of a particular load range, the cyclic J-Integral can be approximated by performing a Modified Monotonic Loading analysis with a single load equal to the cyclic range.

The computed J-Integral will depend greatly on the applied R-Ratio. In this paper, the $M M L$ concept has been demonstrated on a case of unidirectional stress with $R=0$. It is important to note, however, that this technique was originally designed for the case of fully reversed stress with an $\mathrm{R}=-1$ as described by Chen and Chen[21]. It is believed that this concept performs adequately for the case of $\mathrm{R}=-1$ if crack closure is disregarded. However, if crack closure is considered, some differences exist between the cyclic and the MML analyses and so the concept is not felt to be as accurate under these conditions. 


\section{NUMERICAL APPLICATION}

\subsection{Finite Element Model}

The finite element software package, ABAQUS was used for the computational analyses performed in this investigation. Within ABAQUS, a notched industrial test specimen as presented by Leidermark[23] was modelled with appropriate model partitioning employed. Fig 1 shows the geometry of the test specimen used in this investigation. This allowed the implementation of a refined mesh around the most critical region of the notch, whilst a more coarse mesh was modelled in the less critical regions. Second order hexahedral elements were implemented in the model with swept elements in the crack front region and structured elements elsewhere.

A 3D semi-elliptical surface crack with a semi-major axis radius of $1 \mathrm{~mm}$ and a semiminor axis radius of $0.75 \mathrm{~mm}$ was modelled with a focused mesh swept along the crack front to allow for improved accuracy. Cyclic and Modified Monotonic Loading analyses were performed and the results of each compared in order to assess the suitability of the method. Once validated, additional MML analyses were performed to calculate the cyclic J-Integral under uniaxial and out of plane shear loading. The finite element mesh of the test specimen is shown in Fig. 2 and a close-up of the opened crack surfaces shown in Fig. 3.

\subsection{Material Properties and Loading Conditions}

A nickel based super alloy similar to those in turbine applications was used in this investigation. An elastic-perfectly plastic material model was implemented with a Young's Modulus of 207GPa, Poissons' Ratio of 0.29 and Yield Stress of 1000MPa. The accuracy of the technique was tested under uniaxial tension and out of plane shear with both cyclic loading and modified monotonic loading conditions. This allowed the accuracy of the technique to be determined when applied to an industrial test specimen under different loading conditions.

Under uniaxial tension tests, pressure forces were applied to the ends of the specimen. For the cyclic loading analysis, a cyclic load range of 250MPa with a R-Ratio of 
zero was applied and for the equivalent Modified Monotonic Loading analysis, a monotonic pressure load of 250MPa was applied. Under out of plane shear loading, pressure forces were applied to the specimen above and below the notch. For the cyclic loading analysis, a cyclic load range of $600 \mathrm{MPa}$ with a R-Ratio of zero was applied and for the corresponding MML analysis, a monotonic pressure load of 600MPa was applied. Diagrams showing the location of the applied forces for uniaxial tension and out of plane shear are shown in Fig. 4 and Fig. 5 respectively.

These analyses were merely comparative in order to visualise the differences between the cyclic loading and MML concept. Following successful validation, additional tensile tests and out of plane shear tests were performed in order to calculate the cyclic J-Integral using the proposed method.

\section{$5 \quad$ RESULTS AND DISCUSSION}

\subsection{Validation of MML Concept}

The MML results were compared to the cyclic loading analysis to assess the suitability of the technique as a method of calculating the cyclic J-Integral. The stress range and strain range data from the cyclic loading analysis was compared to the stress and strain data of the MML concept. Fig. 6 to Fig. 9 show Von Mises stress and Von Mises stress range and Maximum Principal strain and Maximum Principal strain range data from the MML and cyclic loading analyses for the uniaxial tension test. Fig. 10 to Fig. 13 show Von Mises stress and Von Mises stress range and Maximum Principal strain and Maximum Principal strain range data from the MML and cyclic loading analyses for the out of plane shear test. From comparing the contour plots of the MML and cyclic analyses, it can be seen that the differences between the two are minimal as the contour plots match very closely. As is assumed in Eq. (9) to Eq. (12), if the stress and strain data of the MML concept matches the stress range and strain range data of the cyclic analysis, then the MML can be assumed to be a reasonable approximation of the cyclic J-Integral.

From close inspection of the crack front region, it is noticed that a stress concentration exists at the first contour, where the value is greater than the yield stress. 
This is believed to be due to crack tip singularities and caused by numerical errors in the finite element calculation. In order to maintain the highest level of accuracy, it is important to greatly refine the mesh in the crack front region. However, due to the complexity of the geometry of the model, the degree to which the mesh can be refined is limited. The aim of this investigation is to provide a direct comparison between the stress and strain solutions of the MML concept and cyclic analyses under two different loading conditions. Since identical meshes are used in each analysis, the importance of a highly refined mesh in the critical regions is less critical. For this reason, it is believed that this error has little to no effect on the aim of this research and so the meshing rules used in this investigation are believed to be sufficient. However, it is important to consider the effect of the mesh refinement at the crack front when performing detailed analyses for the calculation of the cyclic J-Integral.

This technique means that a simple monotonic analysis is capable of determining the cyclic J-Integral. This offers a quick and computationally inexpensive method of determining the $\Delta \mathrm{J}$, which would otherwise be difficult and time consuming to calculate. From Eq. (2), it can offer an invaluable method of predicting the EPFM fatigue life of a component.

Some minor discrepancies exist between the contour plots of the MML and the cyclic loading analysis, however these differences are small and so the results are believed to be reasonable. It is felt that the speed and ease of implementation and calculation of this technique far outweighs any potential loss in accuracy.

\subsection{Determination of cyclic J-Integral using MML}

An additional uniaxial tension test and out of plane shear test were performed on the specimen and the induced cyclic J-Integral recorded. The $\Delta \mathrm{J}$ values at different locations along the crack front were monitored as well as the variation of $\Delta \mathrm{J}$ with applied load.

The number of nodes that are defined along the crack front are assigned through the mesh refinement. For this investigation, the employed meshing rules implemented 25 nodes along the circumference of the crack front. Fig. 14 is a schematic diagram of the 
crack front showing the node locations and numbers. Node 1 is located at the far left crack edge and Node 25 is located at the far right crack edge when facing the crack opening. These node numbers are referred to in the results in Table 1, Table 2, Fig. 18 and Fig. 19. For each node along the crack front, the $\Delta \mathrm{J}$ was calculated at 5 contours encircling the crack front. This is illustrated in Fig. 15. The 5 contours at each node were averaged to give a single average value at each node along the crack front. This is believed to be accurate since the J-Integral is a path independent parameter[3], meaning that the value is the same, regardless of the path along which it is calculated. Within ABAQUS, numerical errors due to crack tip singularities mean that the values at each contour are not exactly equal, but any differences are very small and so average values are believed to be sufficiently accurate. Values of the cyclic J-Integral at each contour path at each load are shown in Table 3 for uniaxial tension and Table 4 for out of plane shear loading. It can be seen that the first contour is slightly different to the remaining 4 contours, which have close agreement. This demonstrates the differences that occur at the crack tip due to numerical errors are very minor and so the J-Integral can be assumed to be path independent.

The single values at each node were then tabulated and the average and maximum recorded, as well as far left end of crack, centre of crack and far right end of crack, shown in Table 1 for uniaxial tension, and Table 2 for out of plane shear. The J-Integrals provided in Table 1 and Table 2 are obtained by the traditional elastic plastic fracture mechanics FEA using the MML concept. From the original hypothesis, the $J$ integral calculated from the MML concept is assumed to be equal to the cyclic J-Integral of a cyclic loading analysis when the applied load range is equal to the magnitude of the load in the tables. Therefore these presented values can be regarded as the cyclic J-Integral.

Fig. 16 and Fig. 17 show the cyclic J-Integral variation with increasing uniaxial and out of plane shear load at different locations along the crack front. Under uniaxial loading (Fig. 16), it can be seen that the average, crack left and crack right agree closely due to the symmetry of the loading conditions, however, the maximum value is considerably higher and the crack centre is lower. Upon closer investigation, it was found that the 
variation of the $\Delta \mathrm{J}$ along the crack front varies widely. Fig. 18 shows this for applied uniaxial tension monotonic loads of $250 \mathrm{MPa}$ and $125 \mathrm{MPa}$. A maximum $\Delta \mathrm{J}$ value occurs slightly inside from the end of the crack, 3 nodes from each end and a minimum value occurs in the central region of the crack, where it is relatively constant. However the maximum and minimum values differ by a factor of 3 . The variation of rate of change of cyclic J-Integral with increasing load is not linear across the length of the crack. With increasing load, the maximum $\Delta \mathrm{J}$ values increase more rapidly and the minimum values increase more slowly relative to the values at the centre of the crack.

Under out of plane shear loading (Fig. 17), it can be seen that the J-Integral relationship with increasing load is very different to that of uniaxial tension. Fig. 19 shows the variation of $\Delta \mathrm{J}$ along the crack front under out of plane shear forces of $600 \mathrm{MPa}$ and $300 \mathrm{MPa}$. The maximum cyclic J-Integral coincides with the crack left and crack right positions with the crack centre being considerably less. This variation of $\Delta \mathrm{J}$ is due to the asymmetry of the loading conditions. This implies that the likeliest point of crack initiation under out of plane shear loading is at each side of the crack. The relationship is much more uniform than that of uniaxial tension with the maximum values occurring at the crack edge. The $\Delta \mathrm{J}$ steadily decreases along the crack front to a minimum at the centre with the maximum and minimum values differing by a factor of 5. The rate of change of $\Delta \mathrm{J}$ along the crack front is much more uniform than that of uniaxial tension.

\section{CONCLUSIONS}

This study has proposed and validated the Modified Monotonic Loading concept as a technique for the calculation of the cyclic J-Integral. A cyclic loading analysis was performed on an industrial test specimen under different loading conditions in order to establish a benchmark onto which the MML concept can be validated. The MML concept was then applied to the same test specimen under equivalent monotonic loading. The stress and stress range and strain and strain range data of the MML and cyclic loading analyses were compared, allowing the suitability of the technique to 
calculate the cyclic J-Integral to be ascertained. Once validated, additional analyses were performed on the test specimen which allowed the calculation of the cyclic J-Integral. Its variation with increasing load as well as along the crack front was recorded and the following observations have arisen:

1. Under uniaxial tension, the maximum $\Delta \mathrm{J}$ occurs slightly inside from the crack edge with the minimum being at the centre.

2. Under out of plane shear loading, the maximum $\Delta \mathrm{J}$ occurs at the crack edges, with the minimum being located at the crack centre.

These observed trends in the cyclic J-Integral will be the focus of future investigation to better understand its variation and thus the crack propagation direction. Experimental testing (Fig. 20) has now commenced with the aim of validating the proposed method. This will allow a better understanding of the crack growth behaviour to be gained which will allow assessment of the accuracy of these findings.

This technique allows the three-dimensional crack front detail of the cyclic J-Integral to be monitored and as a result it is possible to identify the likely points of crack propagation, allowing the crack growth path to be predicted. This technique can also be applied to bespoke specimens and is not limited to documented test cases such as compact tension specimens. These features are the main strengths of this concept, making it advantageous to other existing techniques such as the RSM and GE/EPRI methods. This concept is inexpensive both in time and computational power and can be implemented on complex industrial specimens with great ease offering a viable and preferable method of calculating the cyclic J-Integral.

\section{ACKNOWLEDGMENT}

The authors gratefully acknowledge the support of the University of Strathclyde and Siemens Industrial Turbomachinery Ltd during the course of this work. 


\section{REFERENCES}

[1] Irwin, G., 1957, "Analysis of stresses and strains near the end of a crack traversing a plate," Journal of Applied Mechanics, 24, pp. 361-364.

[2] Griffith, A. A., 1921, "The phenomena of rupture and flow in solids," Philosophical Transactions of the Royal Society of London, A 221, pp. 163-198

[3] Rice, J. R., 1968, "A Path Independent Integral and the Approximate Analysis of Strain Concentration by Notches and Cracks," Journal of Applied Mechanics, 35, pp. 379-386

[4] Wells, A. A., 1963, "Application of fracture mechanics at and beyond general yielding," British Welding Journal, 10, pp. 563-70

[5] Broek, D., 1986, "Elementary engineering fracture mechanics", Springer

[6] Anderson, T. L., 2005, "Fracture mechanics: fundamentals and applications," CRC press

[7] R5 Issue 3, June 2003, "Assessment procedure for the high temperature response of structures," EDF Energy

[8] R6 Revision 3, "Assessment of the integrity of structures containing defects," British Energy Generation Ltd, Amendment 10, May 1999

[9] Begley, J. A., Landes, J. D., 1972, "The J-integral as a fracture criterion," ASTM STP 514, pp. 1-20

[10] Kishimoto, K., Aoki, S., Sakata, M., 1980, "On the path independent integral-J," Engineering Fracture Mechanics, 13(4), pp. 841-850

[11] Bucci, R. J., Paris, P. C., Landes, J. D., Rice, J. R., 1972, "J integral estimation procedures," ASTM STP, 514, pp. 40-69

[12] Dowling, N. E., 1976 "Geometry effects and the J-integral approach to elastic-plastic fatigue crack growth," ASTM STP, 601, pp. 19-32.

[13] Zhu, X-K, Joyce, J. A., 2012, "Review of fracture toughness (G, K, J, CTOD, CTOA) testing and standardization," Engineering Fracture Mechanics, 85, pp. 1-46

[14] Dassault Systems Simulia Corp, 2012. Version 6.12-3 
[15] Paris, P., Erdogan, F., 1963, "A critical analysis of crack propagation laws," Journal of Basic Engineering, Transactions of the American Society of Mechanical Engineers, pp. 528-534

[16] Sumpter, J. D. G., Turner, C.E., 1976, "Method for laboratory determination of JC," ASTM STP, 601, pp. 3-18

[17] Dowling, N. E., Begley, J. A., 1976, "Fatigue crack growth during gross plasticity and the J-integral," ASTM-STP, 590, pp. 82-103

[18] Chattopadahyay, J., 2006, "Improved J and COD estimation by GE/EPRI method in elastic to fully plastic transition zone," Engineering Fracture Mechanics, 73, pp. 19591979

[19] Miller, A.G., Ainsworth, R.A., 1989, "Consistency of numerical results for power law hardening materials and the accuracy of the reference stress approximation," Engineering Fracture Mechanics, 32, pp. 233-247

[20] Moës, N., Dolbow, J., Belytschko, T., 1999, "A finite element method for crack growth without remeshing." International Journal of Numerical Methods, 46, pp. 131150

[21] Chen, W., Chen, H., 2013, "Cyclic J-integral using the Linear Matching Method," International Journal of Pressure Vessels and Piping, 108-109, pp. 72-80

[22] K. Tanaka, 1983, "The cyclic J-integral as a criterion for fatigue crack growth," International Journal of Fracture, 22, pp. 91-104

[23] Leidermark, D.,Moverare, J., Simonsson, K., Sjöström, S., 2011, “A combined critical plane and critical distance approach for predicting fatigue crack initiation in notched single-crystal superalloy components," International Journal of Fatigue, 33(10), pp. 1351-1359 


\section{Figure Captions List}

Fig. 1 The geometry of the investigated test specimens

Fig. 2 Finite element mesh of structure showing (a) entire specimen and close up view of notch (b) and (c)

Fig. 3 Open crack surfaces

Fig. $4 \quad$ Location of applied loads under uniaxial tension conditions

Fig. $5 \quad$ Location of applied loads under out of plane shear conditions

Fig. 6 Contour plots of (a) stress from MML analysis and (b) stress range from cyclic loading analysis under uniaxial loading conditions

Fig. 7 Enlarged view of contour plots of crack tip of (a) stress from MML analysis and (b) stress range from cyclic loading analysis under uniaxial loading conditions

Fig. 8 Contour plots of (a) strain from MML analysis and (b) strain range from cyclic loading analysis under uniaxial loading conditions

Fig. 9 Enlarged view of contour plots of crack tip of (a) strain from MML analysis and (b) strain range from cyclic loading analysis under uniaxial loading conditions

Fig. 10 Contour plots of (a) stress from MML analysis and (b) stress range from cyclic loading analysis under out of plane shear loading conditions

Fig. 11 Enlarged view of contour plots of crack front of (a) stress from MML 
analysis and (b) stress range from cyclic loading analysis under out of plane shear loading conditions

Fig. 12 Contour plots of (a) strain from MML analysis and (b) strain range from cyclic loading analysis under out of plane shear loading conditions

Fig. 13 Enlarged view of contour plots of crack front of (a) strain from MML analysis and (b) strain range from cyclic loading analysis under out of plane shear loading conditions

Fig. 14 Schematic diagram showing node numbering

Fig. 15 Enlarged view of crack tip showing contour paths

Fig. $16 \Delta$-integral variation with increasing uniaxial load

Fig. $17 \Delta$-integral variation with increasing out of plane shear load

Fig. $18 \Delta$ J variation along crack front under uniaxial tension loading conditions

Fig. $19 \Delta \mathrm{J}$ variation along crack front under out of plane shear loading conditions

Fig. 20 Image of experimental setup, showing (a) specimen in tensile test machine and (b) enlarged view of specimen positioned in machine with extensometers positioned on specimen edge

\section{Table Caption List}

Table $1 \quad J$-integral variation with increasing uniaxial load

Table 2 J-integral variation with increasing out of plane shear load 
Table 3 The Variation Of The Cyclic J-Integral At Each Contour Path Under Uni-Axial Tensile Loading

Table 4 The Variation Of The Cyclic J-Integral At Each Contour Path Under Out-Of-Plane Shear Loading 


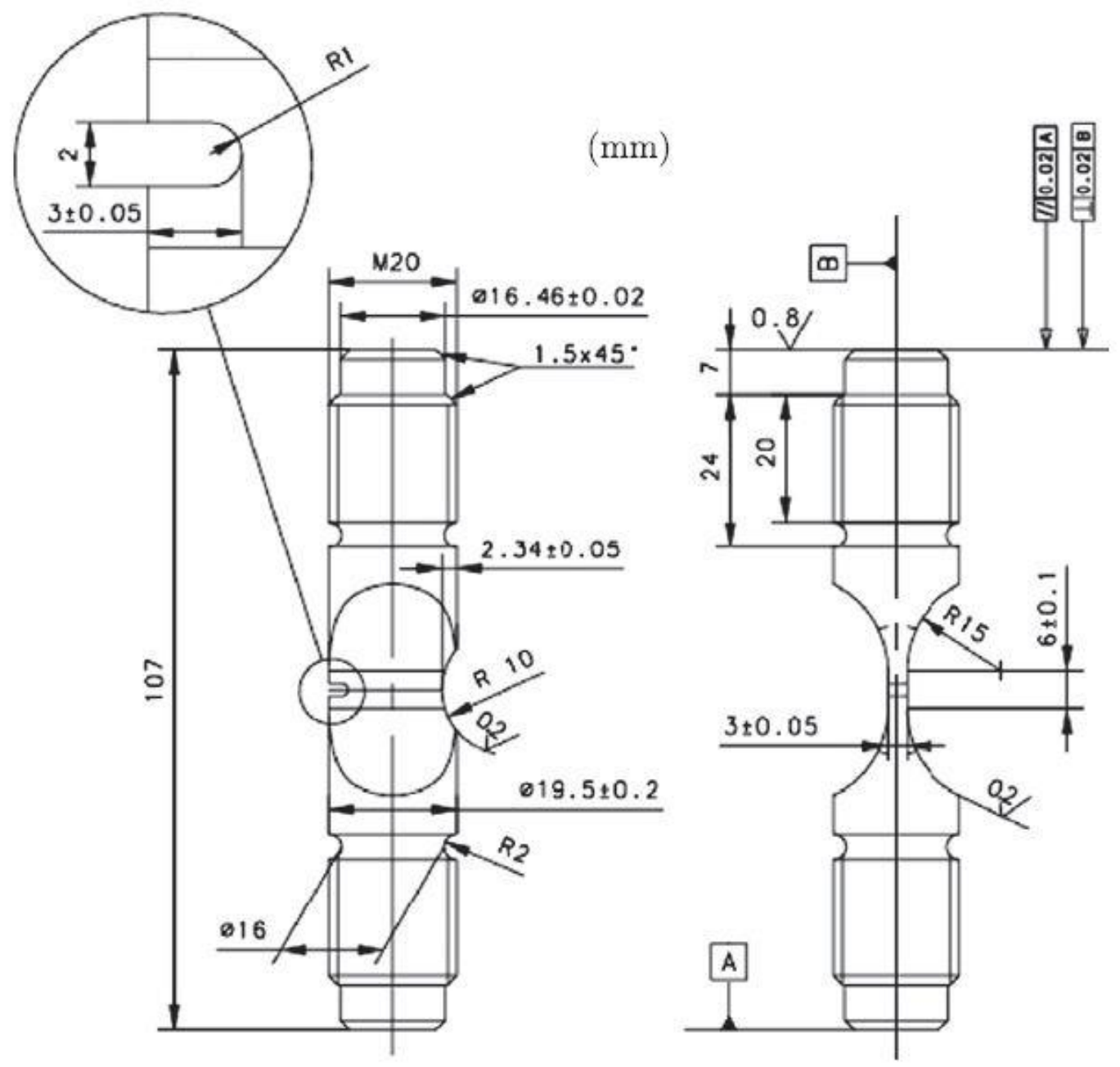

Fig 1: The geometry of the investigated test specimens [23] 

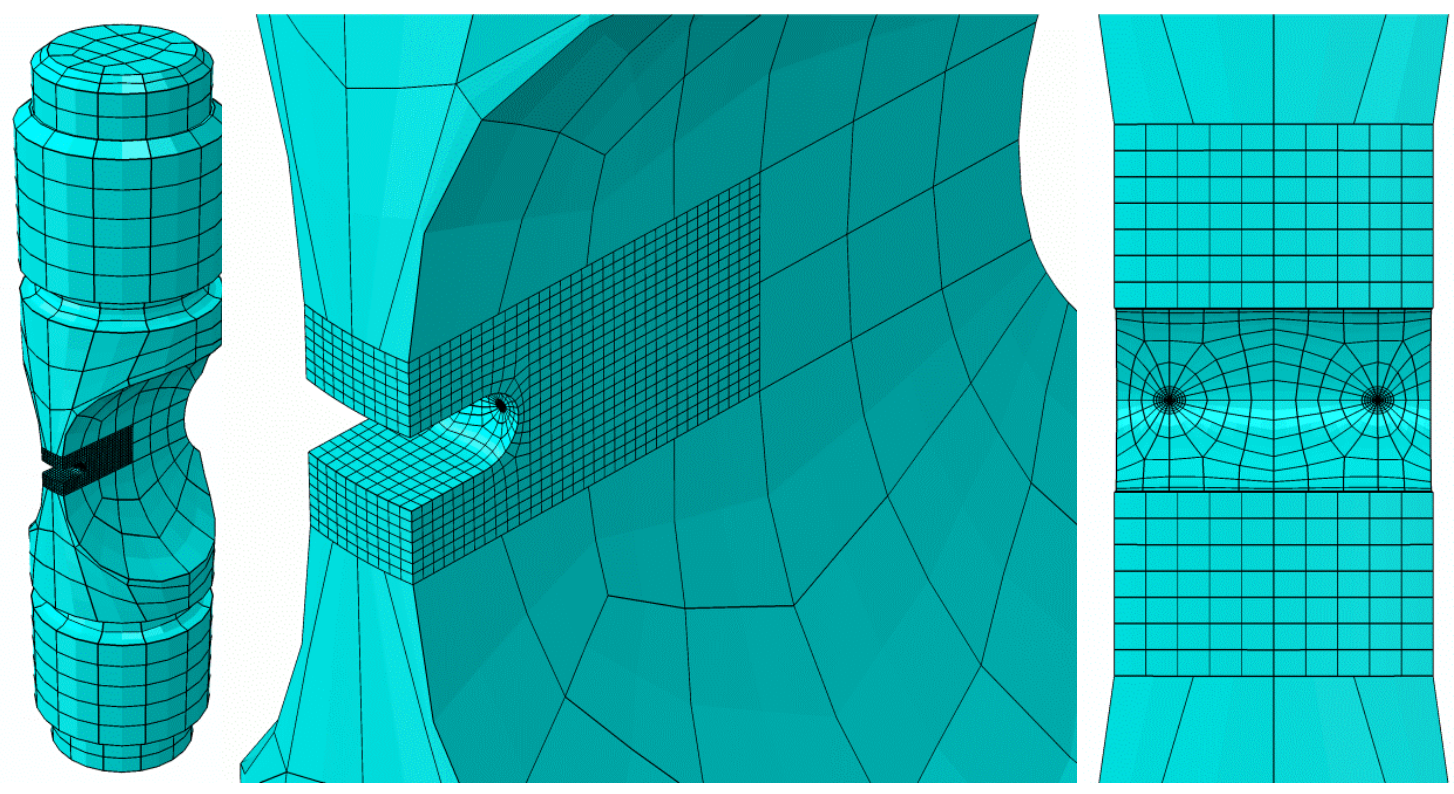

Fig. 2: Finite element mesh of structure showing (a) entire specimen and close up view of notch (b) and (c) 


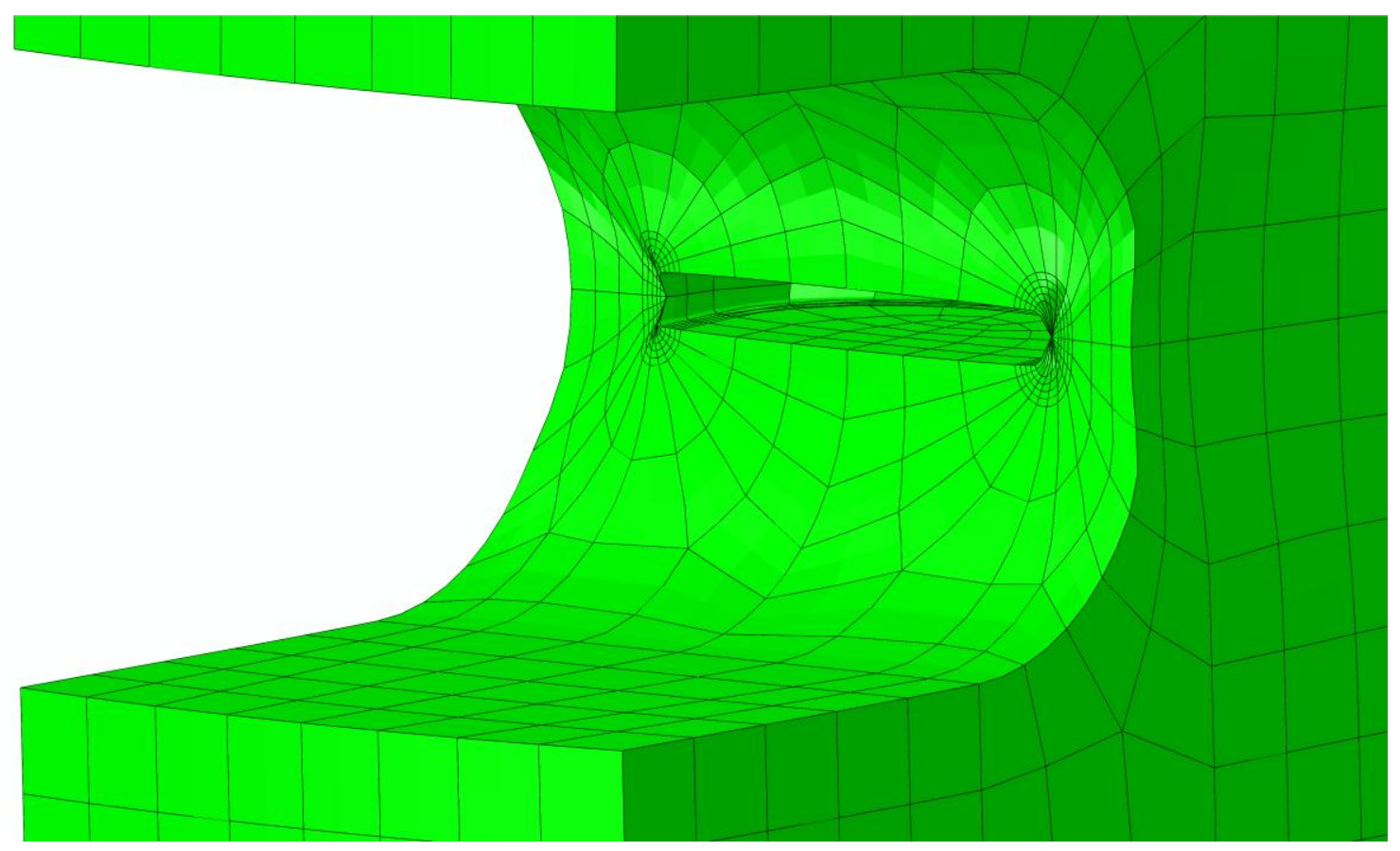

Fig. 3: Open crack surfaces 

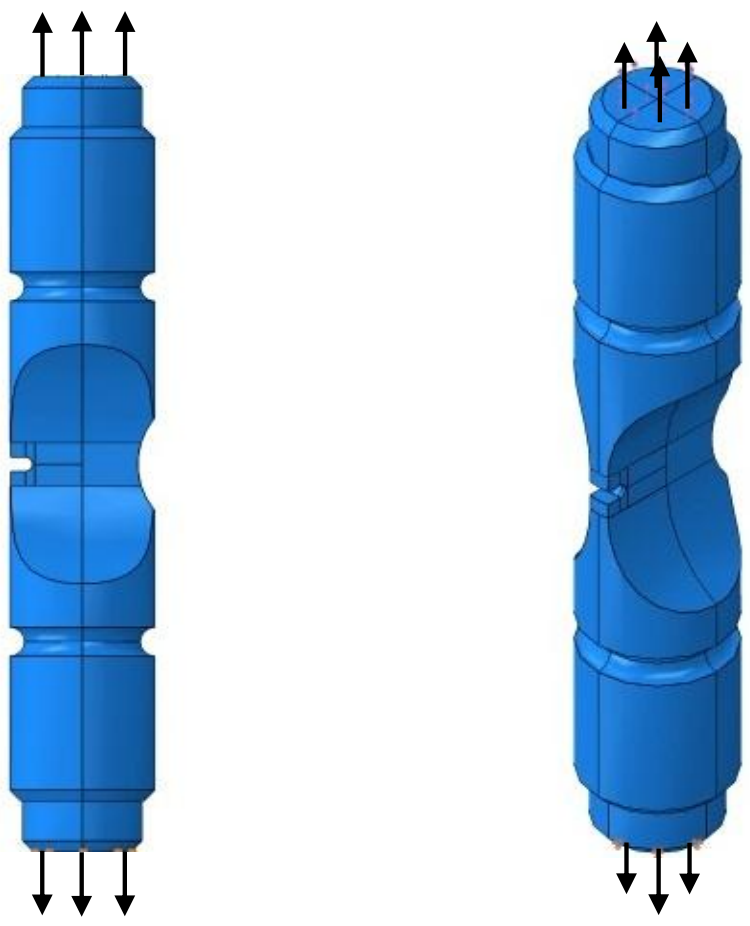

Fig. 4: Location of applied loads under uniaxial tension conditions 

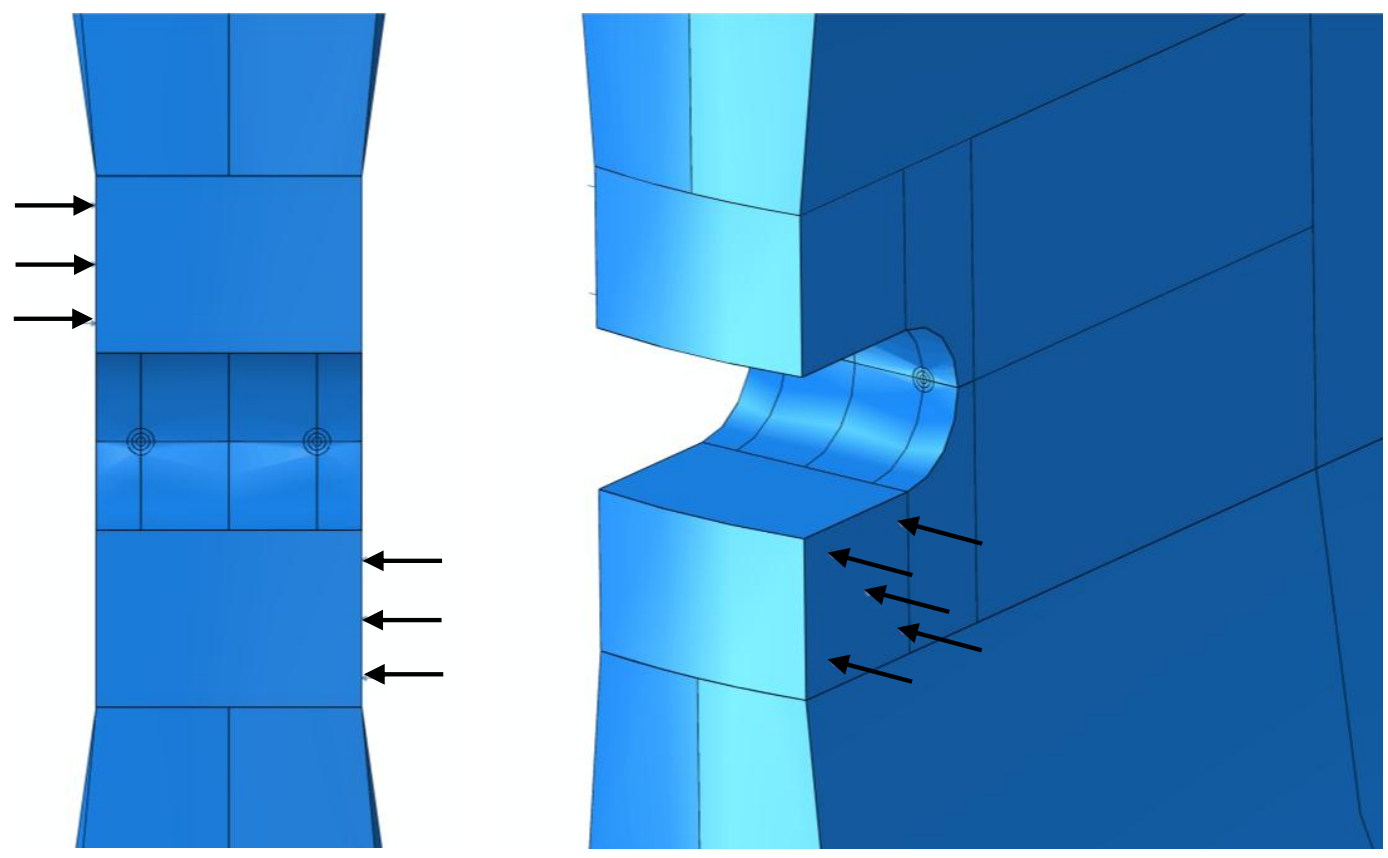

Fig. 5: Location of applied loads under out of plane shear conditions 

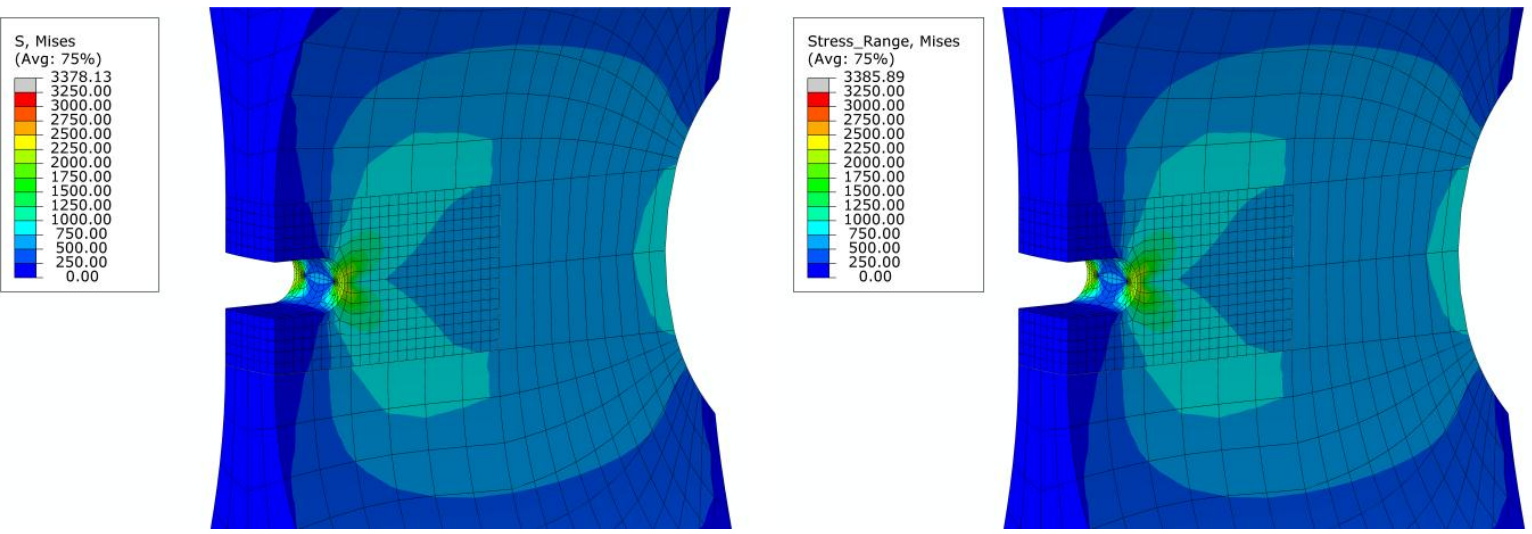

Fig. 6: Contour plots of (a) Von Mises stress from MML analysis and (b) Von Mises stress range from cyclic loading analysis under uniaxial loading conditions 

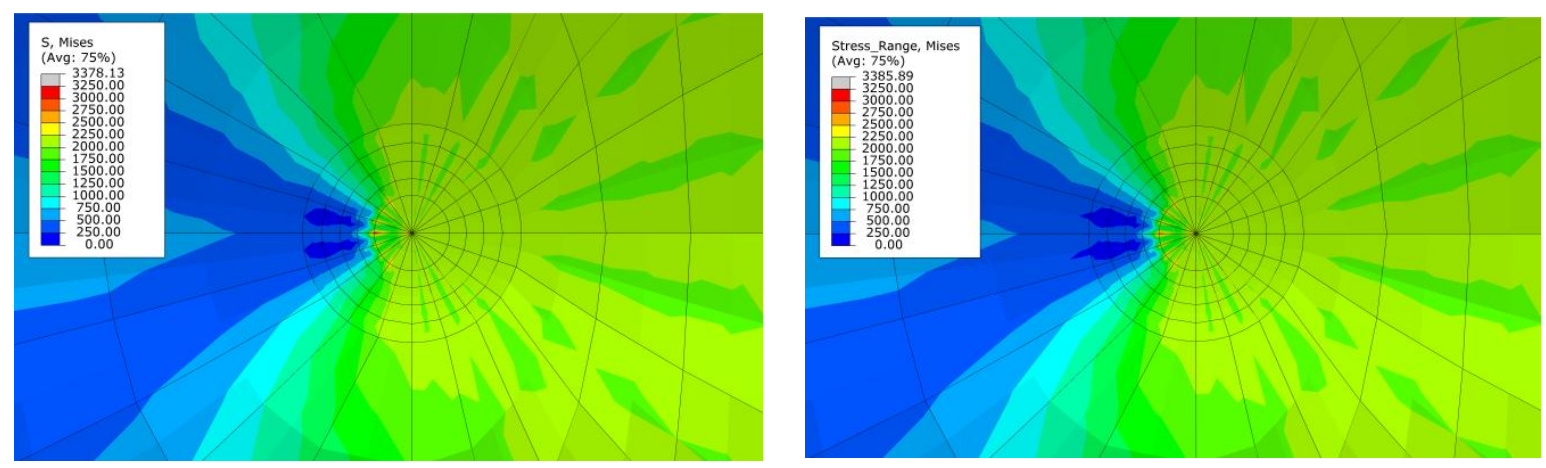

Fig. 7: Enlarged view of contour plots of crack tip of (a) Von Mises stress from MML analysis and (b) Von Mises stress range from cyclic loading analysis under uniaxial loading conditions 

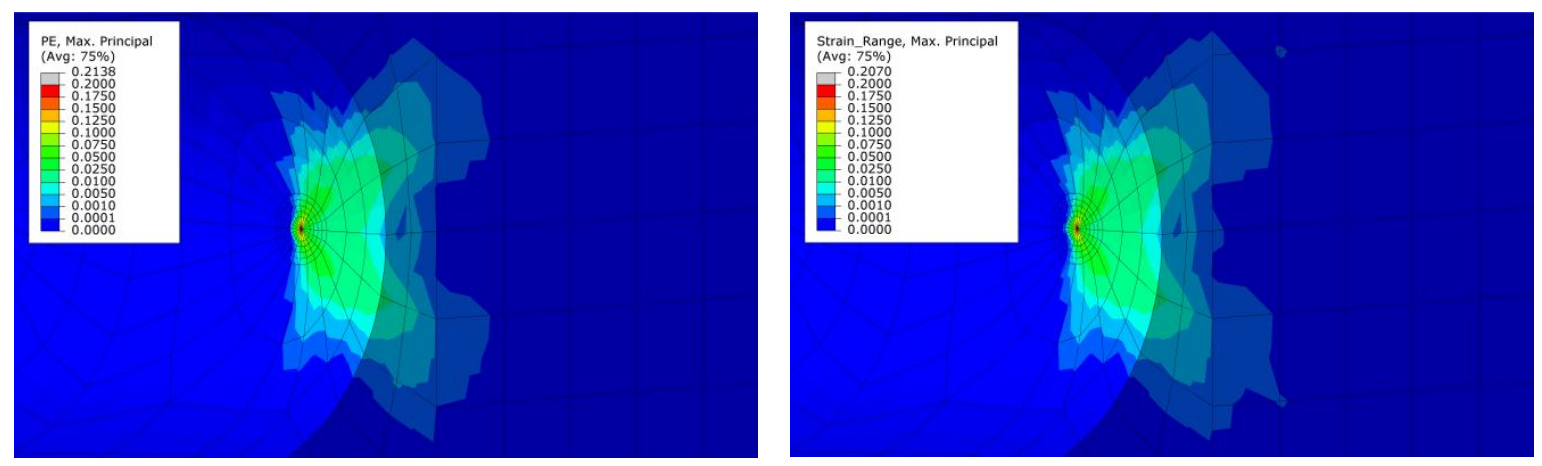

Fig. 8: Contour plots of (a) Maximum Principal strain from MML analysis and (b) Maximum Principal strain range from cyclic loading analysis under uniaxial loading conditions 

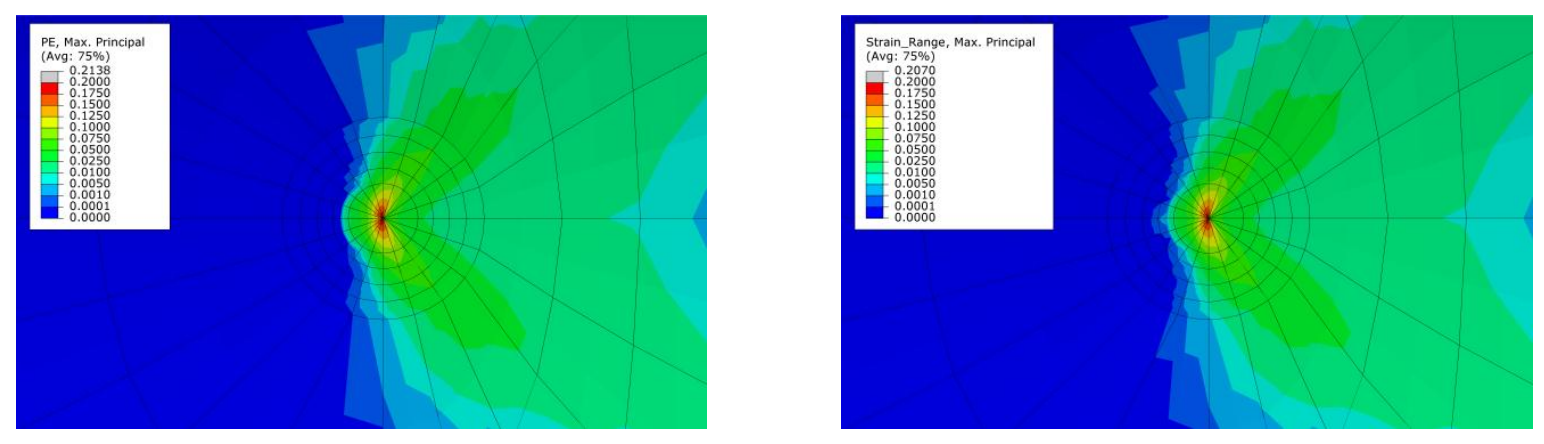

Fig. 9: Enlarged view of contour plots of crack tip of (a) Maximum Principal strain from MML analysis and (b) Maximum Principal strain range from cyclic loading analysis under uniaxial loading conditions 

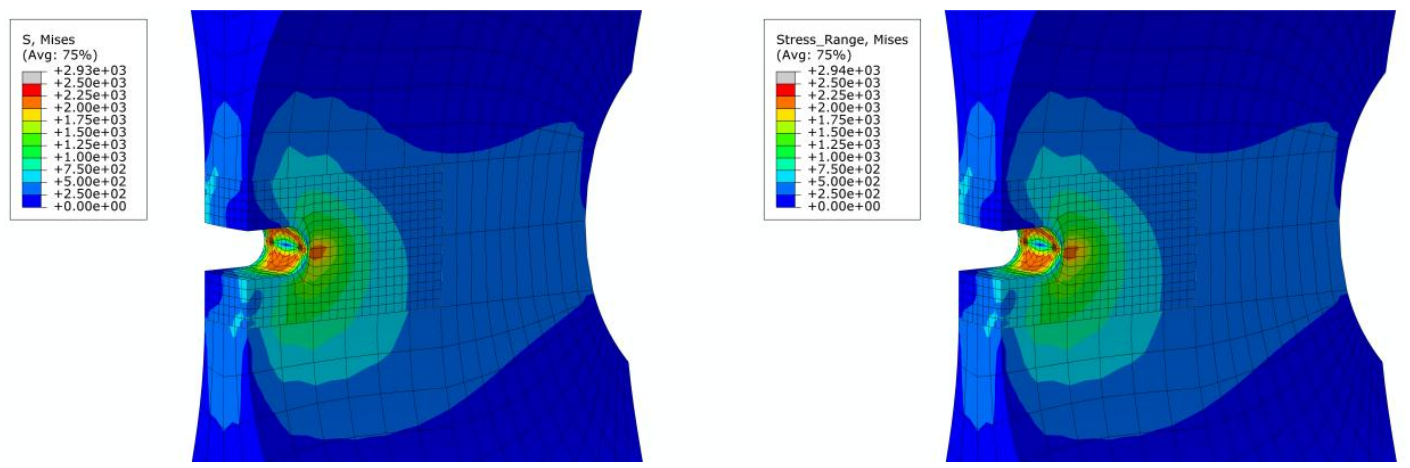

Fig. 10: Contour plots of (a) Von Mises stress from MML analysis and (b) Von Mises stress range from cyclic loading analysis under out of plane shear loading conditions 

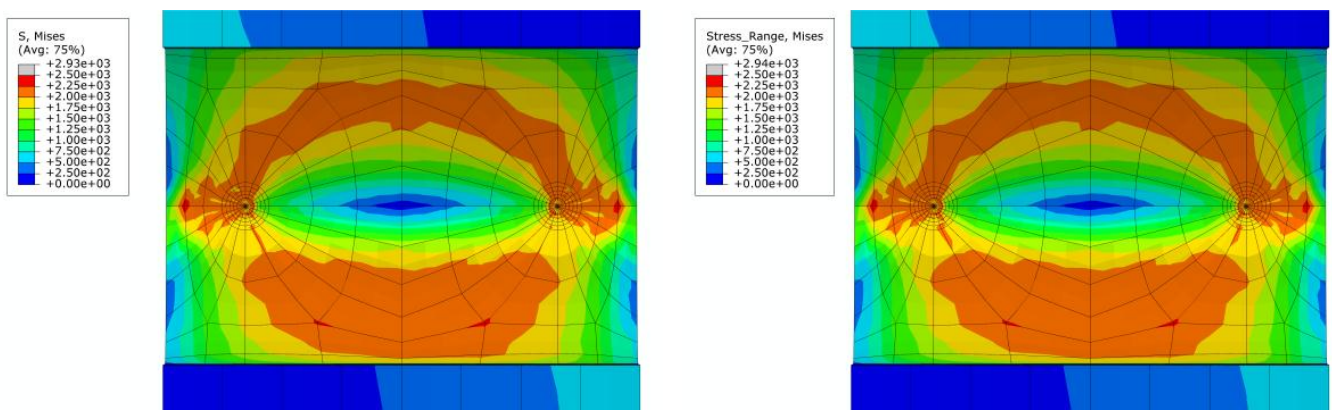

Fig. 11: Enlarged view of contour plots of crack front of (a) Von Mises stress from MML analysis and (b) Von Mises stress range from cyclic loading analysis under out of plane shear loading conditions 


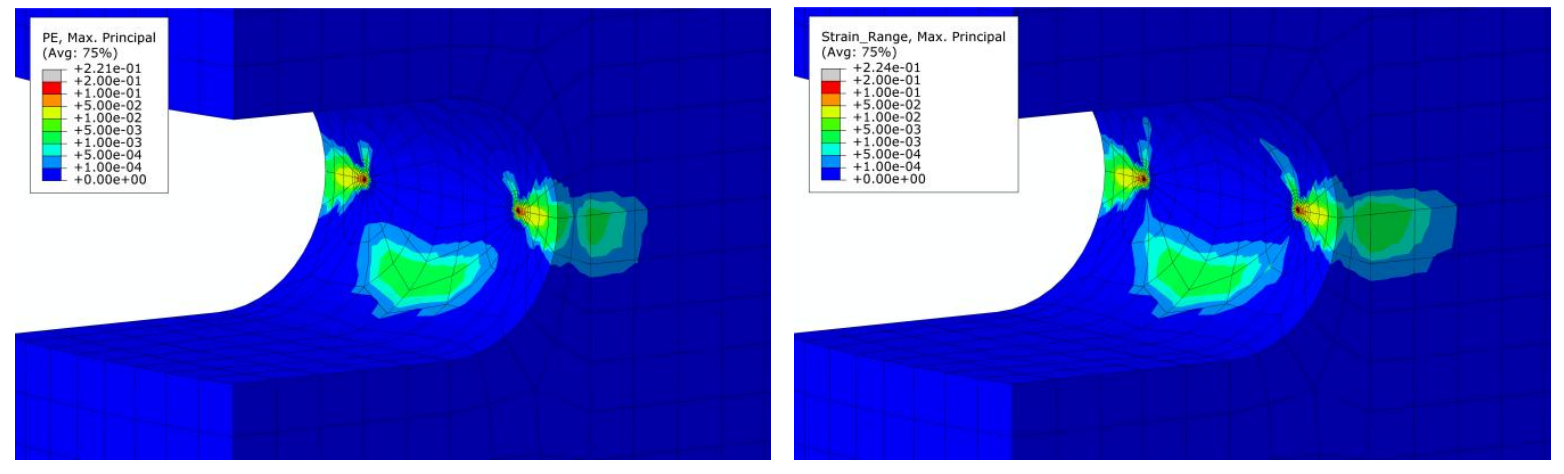

Fig. 12: Contour plots of (a) Maximum Principal strain from MML analysis and (b) Maximum Principal strain range from cyclic loading analysis under out of plane shear loading conditions 

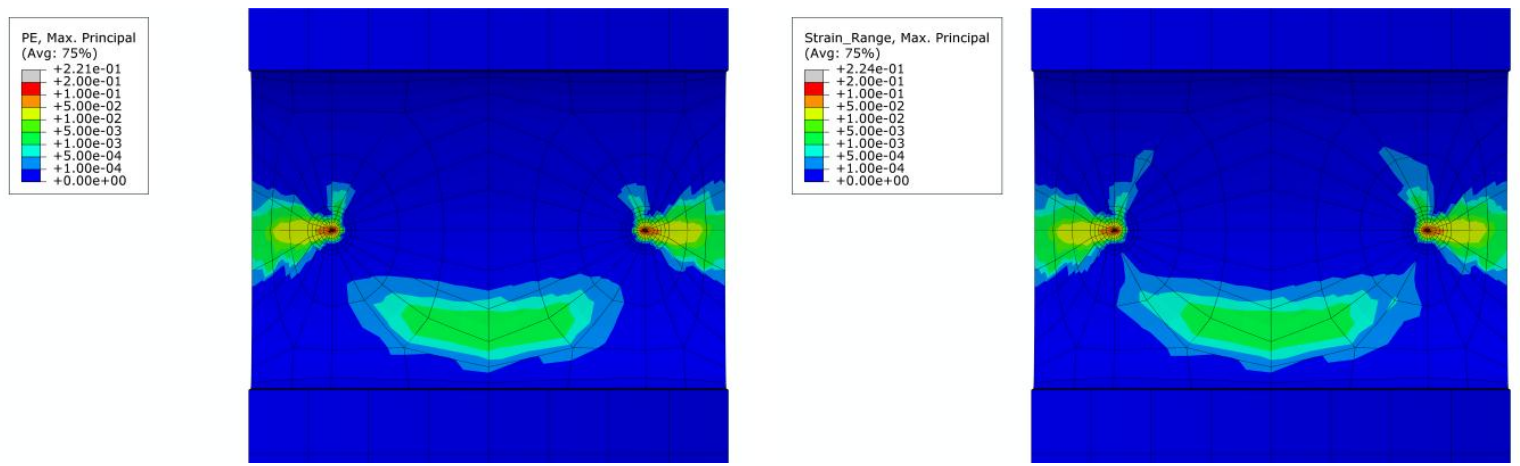

Fig. 13: Enlarged view of contour plots of crack front of (a) Maximum Principal strain from MML analysis and (b) Maximum Principal strain range from cyclic loading analysis under out of plane shear loading conditions 


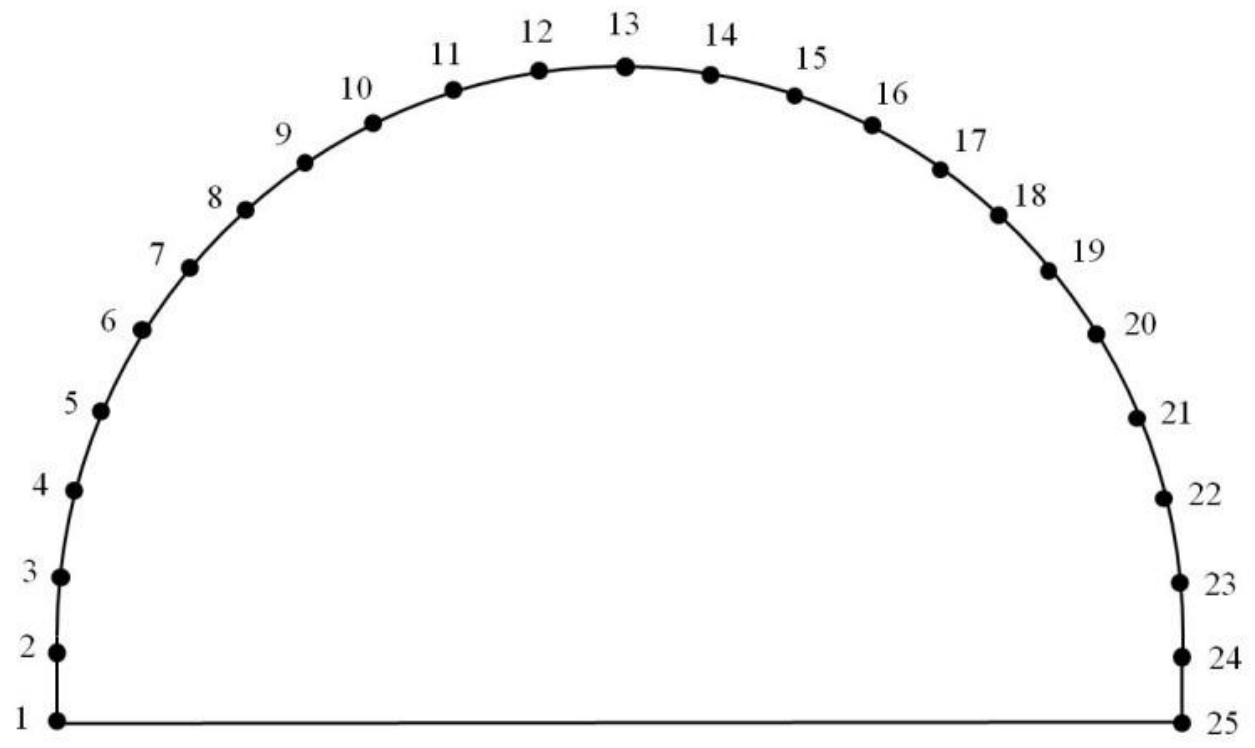

Fig. 14: Schematic diagram showing node numbering 


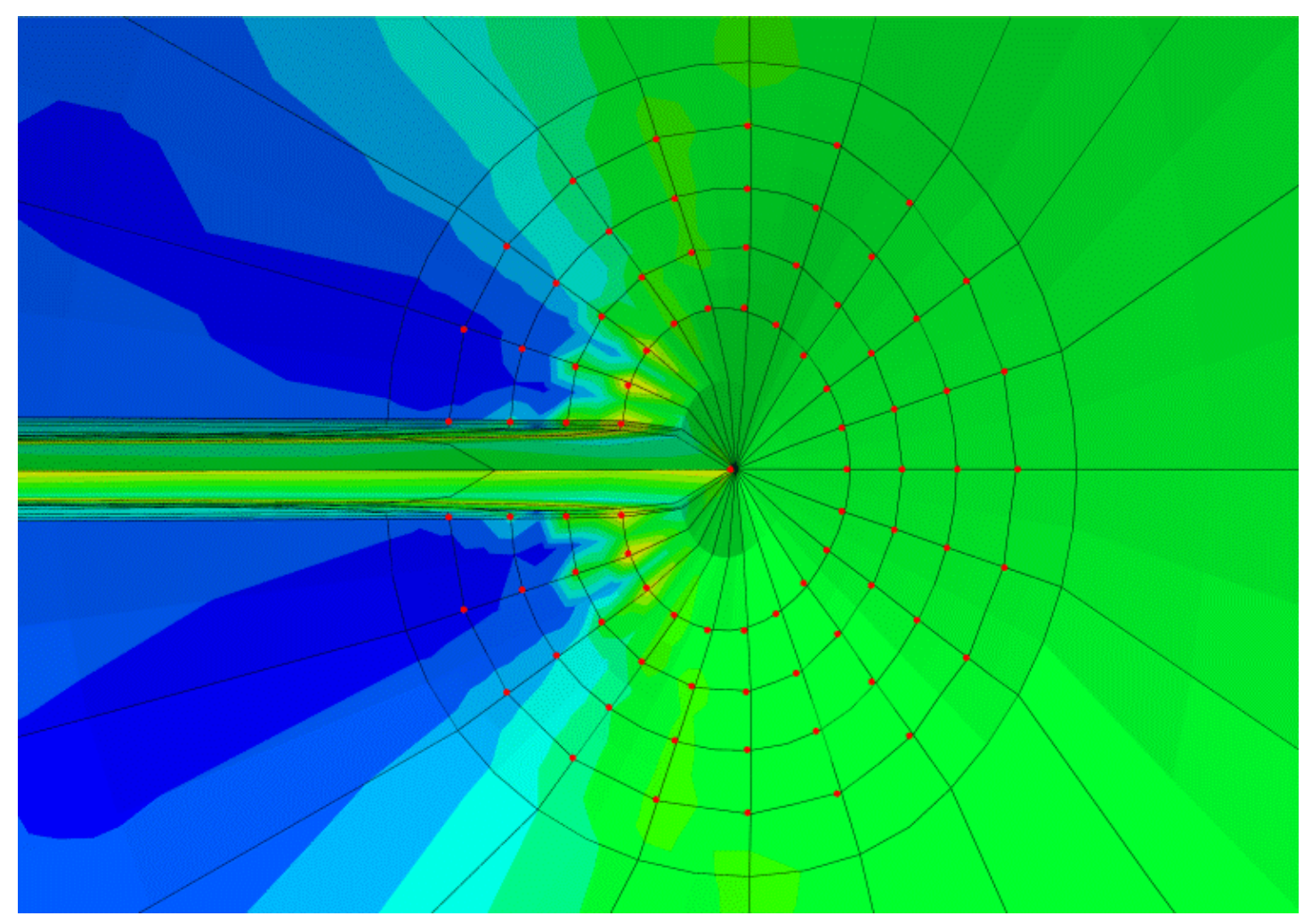

Fig. 15: Enlarged view of crack tip showing contour paths 


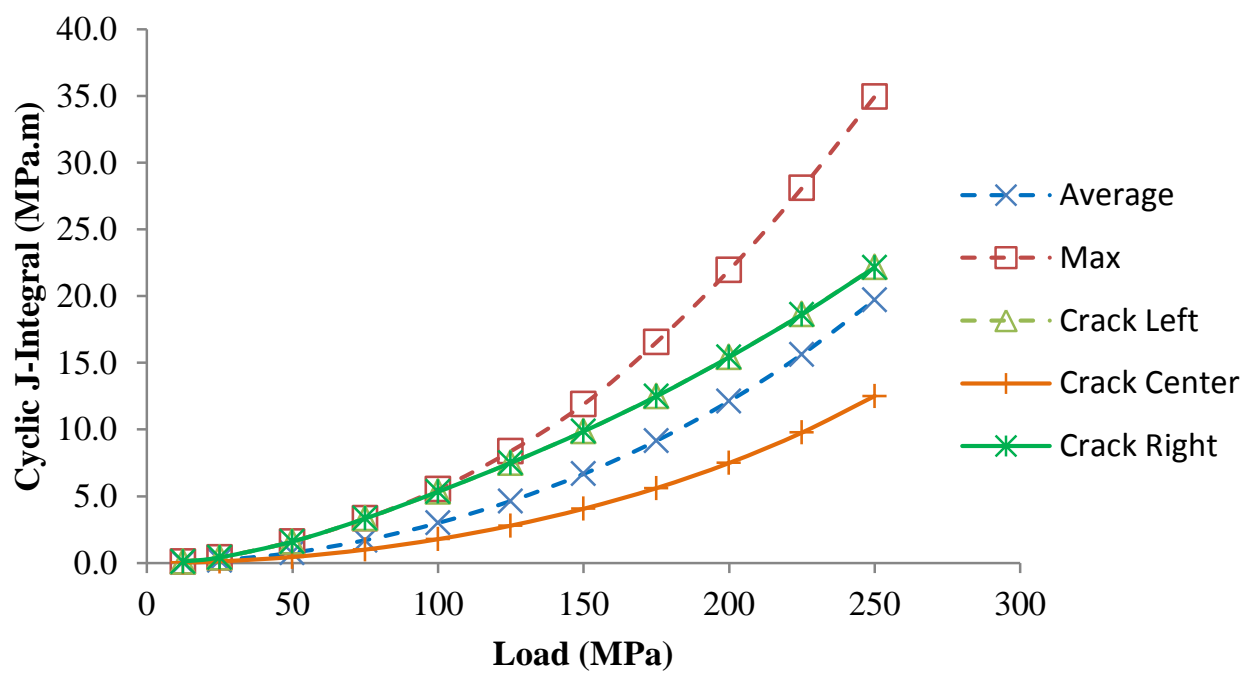

Fig. 16: $\Delta \mathrm{J}$-integral variation with increasing uniaxial load 


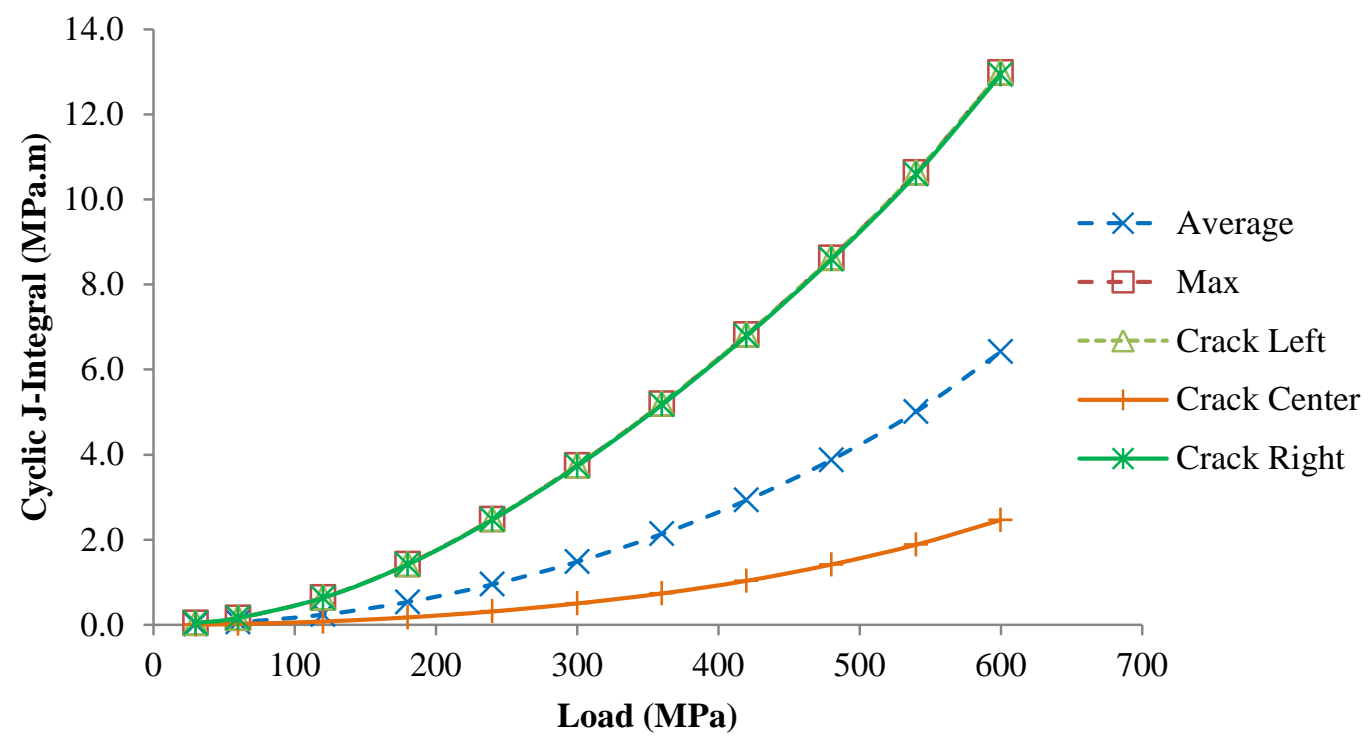

Fig. 17: $\Delta \mathrm{J}$-integral variation with increasing out of plane shear load 


\section{Cyclic J-Integral Variation Along Crack Front}

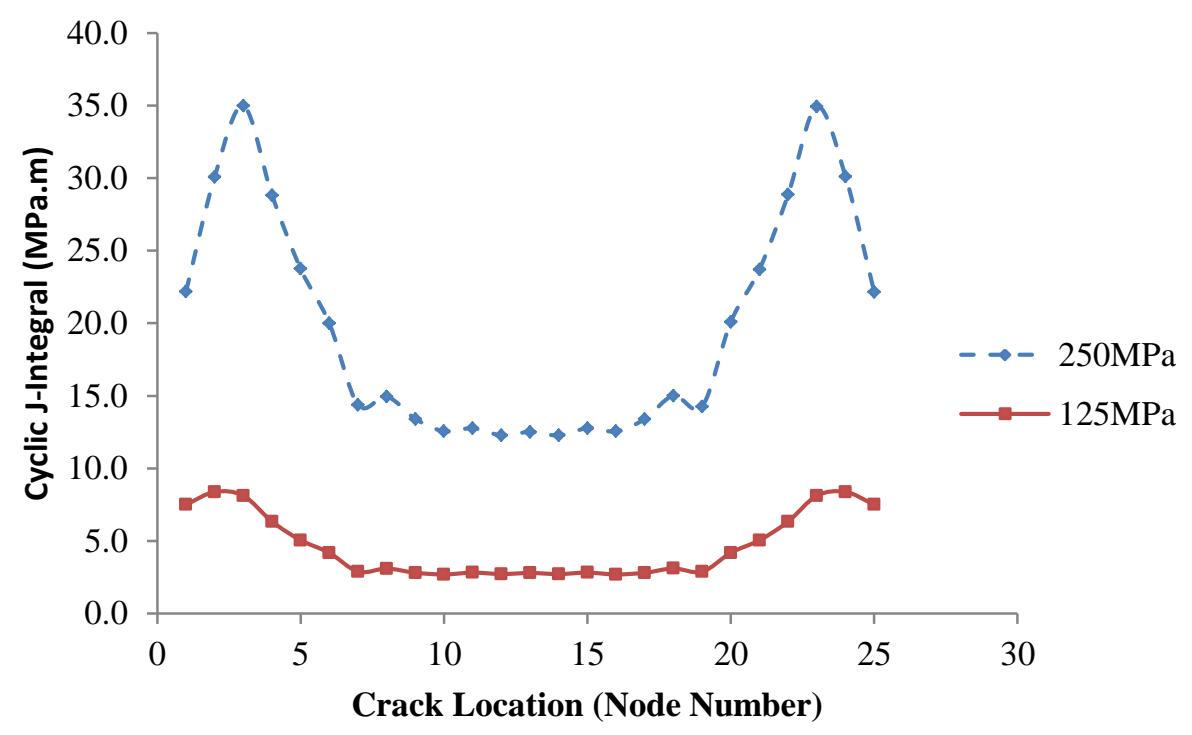

Fig. 18: $\Delta \mathrm{J}$ variation along crack front under uniaxial tension loading conditions 


\section{Cyclic J-Integral Variation Along Crack Front}

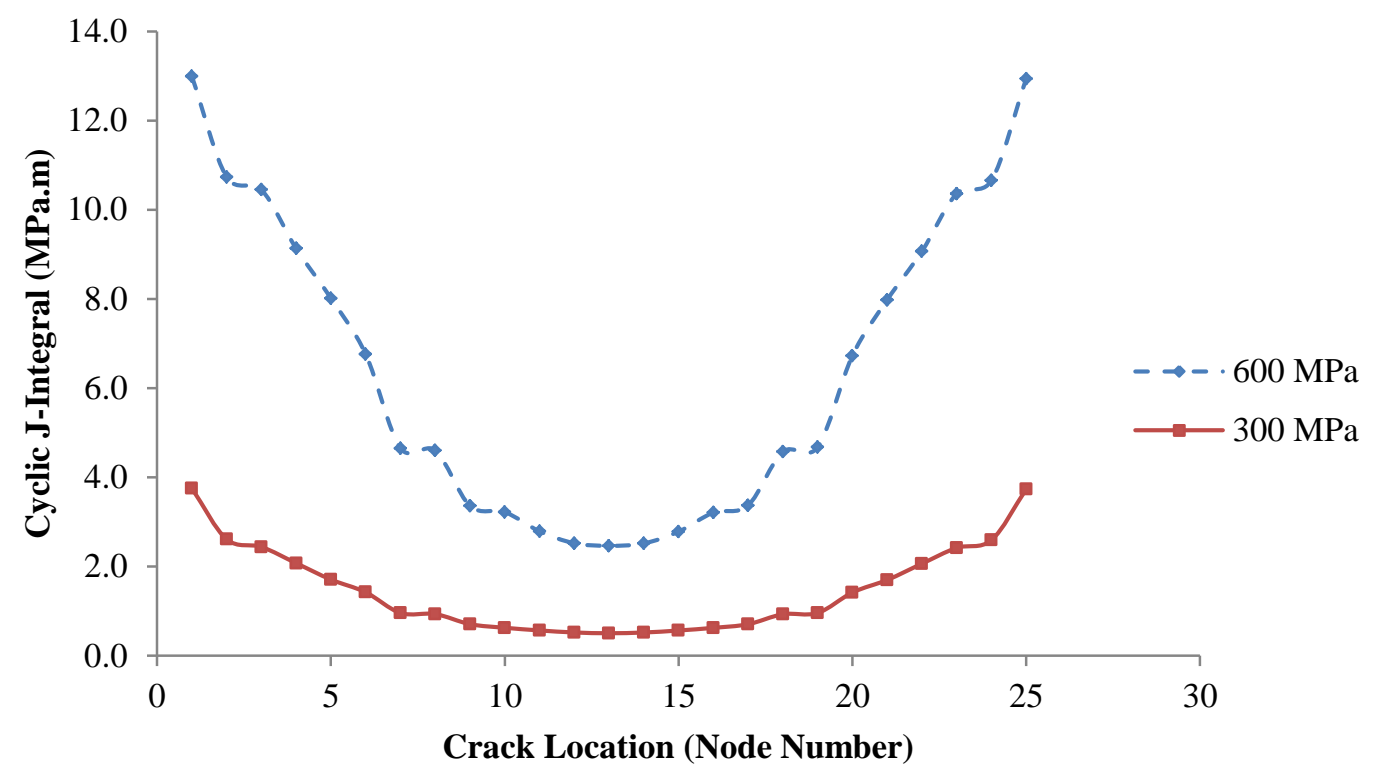

Fig. 19: $\Delta \mathrm{J}$ variation along crack front under out of plane shear loading conditions 


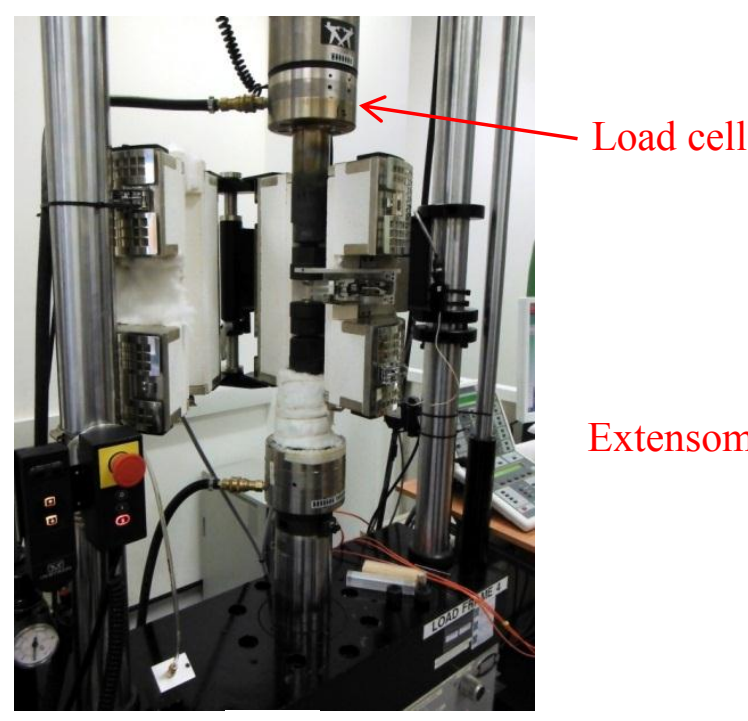

(a)

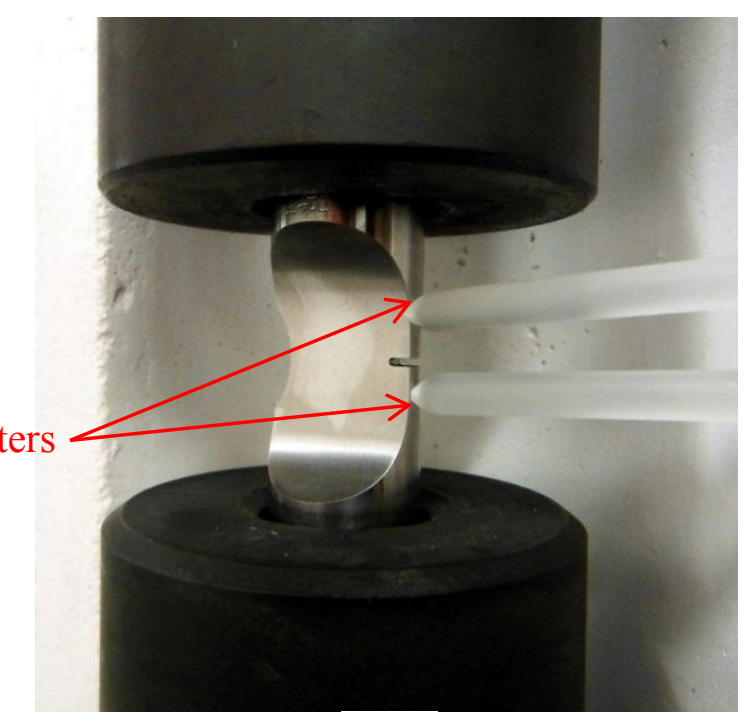

(b)

Fig. 20: Image of experimental setup, showing (a) specimen in tensile test machine and (b) enlarged view of specimen positioned in machine with extensometers positioned on specimen edge 
Table 1: J-integral variation with increasing uniaxial load

\begin{tabular}{|c|c|c|c|c|c|}
\hline \multirow{2}{*}{$\begin{array}{c}\text { Load } \\
(\mathrm{MPa})\end{array}$} & Average & Max & $\begin{array}{c}\text { Crack Left } \\
\text { (Node 1) }\end{array}$ & $\begin{array}{c}\text { Crack } \\
\text { Centre } \\
\text { (Node 13) }\end{array}$ & $\begin{array}{c}\text { Crack Right } \\
\text { (Node 25) }\end{array}$ \\
\cline { 2 - 6 } & 0.0478 & 0.1023 & 0.1022 & 0.0276 & 0.1023 \\
\hline 12.5 & 0.1912 & 0.4090 & 0.4088 & 0.1105 & 0.4090 \\
\hline 25 & 0.7631 & 1.6015 & 1.6008 & 0.4422 & 1.6015 \\
\hline 50 & 1.7005 & 3.3309 & 3.3298 & 0.9972 & 3.3309 \\
\hline 75 & 2.9941 & 5.5183 & 5.3354 & 1.7783 & 5.3370 \\
\hline 100 & 4.6408 & 8.3802 & 7.5034 & 2.7918 & 7.5046 \\
\hline 125 & 6.6734 & 11.8929 & 9.8692 & 4.0571 & 9.8653 \\
\hline 150 & 9.1552 & 16.5454 & 12.5029 & 5.6038 & 12.4929 \\
\hline 175 & 12.1294 & 21.9518 & 15.4460 & 7.4913 & 15.4342 \\
\hline 200 & 15.6357 & 28.1072 & 18.6470 & 9.7715 & 18.6323 \\
\hline 225 & 19.7118 & 34.9707 & 22.1816 & 12.5141 & 22.1594 \\
\hline 250 & \multicolumn{7}{|c|}{} \\
\hline
\end{tabular}


Table 2: J-integral variation with increasing out of plane shear load

\begin{tabular}{|c|c|c|c|c|c|}
\hline \multirow{2}{*}{$\begin{array}{c}\text { Load } \\
(\mathrm{MPa})\end{array}$} & Average & Max & $\begin{array}{c}\text { Jrack Left } \\
\text { (Node 1) }\end{array}$ & $\begin{array}{c}\text { Crack } \\
\text { Centre } \\
\text { (Node 13) }\end{array}$ & $\begin{array}{c}\text { Crack Right } \\
\text { (Node 25) }\end{array}$ \\
\hline 30 & 0.0149 & 0.0405 & 0.0405 & 0.0048 & 0.0402 \\
\hline 60 & 0.0596 & 0.1619 & 0.1619 & 0.0191 & 0.1609 \\
\hline 120 & 0.2378 & 0.6376 & 0.6376 & 0.0765 & 0.6335 \\
\hline 180 & 0.5347 & 1.4277 & 1.4277 & 0.1737 & 1.4190 \\
\hline 240 & 0.9501 & 2.4856 & 2.4856 & 0.3133 & 2.4715 \\
\hline 300 & 1.4805 & 3.7501 & 3.7501 & 0.5024 & 3.7321 \\
\hline 360 & 2.1367 & 5.1968 & 5.1968 & 0.7363 & 5.1731 \\
\hline 420 & 2.9299 & 6.8236 & 6.8236 & 1.0339 & 6.7934 \\
\hline 480 & 3.8784 & 8.6282 & 8.6282 & 1.4173 & 8.5911 \\
\hline 540 & 5.0106 & 10.6364 & 10.6364 & 1.8828 & 10.5925 \\
\hline 600 & 6.4210 & 12.9883 & 12.9883 & 2.4636 & 12.9380 \\
\hline
\end{tabular}


Table 3: The Variation Of The Cyclic J-Integral At Each Contour Path Under Uni-Axial Tensile Loading UNI-AXIAL TENSION

Average Cyclic J-Integral at Contours 1 to 5 (Averaged from all 25 Nodes)

\begin{tabular}{|c|c|c|c|c|}
\hline & \multicolumn{3}{|c|}{ Load (MPa) } \\
\hline & & 25 & 125 & 250 \\
\hline \multirow{5}{*}{ 总 } & 1 & 0.184621 & 4.251451 & 17.03563 \\
\hline & 2 & 0.192854 & 4.733544 & 20.19799 \\
\hline & 3 & 0.192909 & 4.740572 & 20.39367 \\
\hline & 4 & 0.192873 & 4.73978 & 20.45251 \\
\hline & 5 & 0.192776 & 4.738423 & 20.47896 \\
\hline
\end{tabular}

Maximum Cyclic J-Integral at Contours 1 to 5 (Maximum of all 25 Nodes)

\begin{tabular}{|c|c|c|c|c|}
\hline \multicolumn{2}{|c|}{} & \multicolumn{3}{c|}{ Load (MPa) } \\
\cline { 3 - 5 } \multicolumn{2}{|c|}{} & 25 & 125 & 250 \\
\hline \multirow{3}{*}{$\Xi$} & 1 & 0.392916 & 7.49406 & 29.666 \\
\cline { 2 - 5 } & 2 & 0.414454 & 8.54818 & 35.9647 \\
\cline { 2 - 5 } & 3 & 0.413202 & 8.60067 & 36.4048 \\
\cline { 2 - 5 } & 4 & 0.412717 & 8.62294 & 36.4568 \\
\cline { 2 - 5 } & 5 & 0.411896 & 8.63546 & 36.4538 \\
\hline
\end{tabular}


Table 4: The Variation Of The Cyclic J-Integral At Each Contour Path Under Out-Of-Plane Shear Loading

\section{OUT-OF-PLANE SHEAR}

Average Cyclic J-Integral at Contours 1 to 5 (Averaged from all 25 Nodes)

\begin{tabular}{|c|c|c|c|c|}
\hline & \multicolumn{3}{|c|}{ Load (MPa) } \\
\hline & & 60 & 300 & 600 \\
\hline \multirow{5}{*}{ 节 } & 1 & 0.057376 & 1.342511 & 5.574633 \\
\hline & 2 & 0.060203 & 1.512485 & 6.611787 \\
\hline & 3 & 0.060199 & 1.51548 & 6.644833 \\
\hline & 4 & 0.060197 & 1.516003 & 6.638904 \\
\hline & 5 & 0.060185 & 1.515926 & 6.634665 \\
\hline
\end{tabular}

Maximum Cyclic J-Integral at Contours 1 to 5 (Maximum of all 25 Nodes)

\begin{tabular}{|c|c|c|c|c|}
\hline \multicolumn{2}{|c|}{} & \multicolumn{3}{c|}{ Load (MPa) } \\
\cline { 3 - 5 } \multicolumn{2}{|c|}{} & 60 & 300 & 600 \\
\hline \multirow{4}{*}{$\Xi$} & 1 & 0.152483 & 3.2396 & 10.9881 \\
\cline { 2 - 5 } & 2 & 0.164193 & 3.8566 & 13.4225 \\
\cline { 2 - 5 } & 3 & 0.164206 & 3.88042 & 13.545 \\
\cline { 2 - 5 } & 4 & 0.164372 & 3.88744 & 13.5083 \\
\cline { 2 - 5 } & 5 & 0.164298 & 3.88661 & 13.4778 \\
\hline
\end{tabular}

